\title{
Factores que influyen en la intensidad de los síntomas del tracto urinario inferior en pacientes con hiperplasia benigna de próstata
}

\author{
Omar Díaz-Cucho,a, María Aparcana-Huayanca ${ }^{1, a}$, Angel Morales-Alvarez, \\ Facultad de Medicina, Universidad Nacional San Luis Gonzaga. Ica, Perú1 \\ Médico cirujanoa
}

\begin{abstract}
O bjetivo: Determinar los factores que influyen en la intensidad de los síntomas del tracto urinario inferior (STUI) en pacientes con hiperplasia benigna de próstata en un hospital del Perú. Materiales y métodos: Se realizó un estudio transversal durante el año 2011 en 382 varones atendidos en el Hospital IV “Augusto Hernández Mendoza" de Ica. Mediante encuesta se recogió información sociodemográfica y se determinó la intensidad de STUI mediante el International Prostate Symptoms Score. Se aplicó chi cuadrado $\left(\mathrm{X}^{2}\right)$ para valorar asociación entre variables cualitativas y se estimó el odds ratio (OR). Se realizó regresión logística para evaluar la probabilidad de tener STUI moderados/severos. Resultados: La prevalencia de STUI moderados/severos fue del 85,1\%. El 41,1\% eran diabéticos y el 80,4\% eran hipertensos. La edad mayor o igual a 60 años, frente a edad menor de 60 años, presentó 2,63 veces más frecuencia de STUI moderados/severos(IC95\%1,35-5,09), así como el nivel bajo de estudios, respecto al alto, (OR 2,45; IC95\% 1,06-5,61), la diabetes (OR 2,17; IC95\% 1,15-4,07), la hipertensión (OR 2,4; IC95\% 1,294,47 ), la ingesta excesiva de alcohol al menos una vez al mes (OR 4,44; IC $95 \% 2,47-7,99$ ) y el consumo de al menos un cigarrillo diario (OR 3,42; IC95\% 1,87-6,22). La prostatitis se asoció a una menor prevalencia de STUI moderados/severos (OR 0,36; IC95\% 0,20-0,66). Conclusiones: La edad, el nivel bajo de estudios, la hipertensión, la diabetes, el consumo de alcohol y cigarrillos se asociaron con STUI más severos.
\end{abstract}

Palabras clave: Hiperplasia prostática, tracto urinario, consumo de bebidas alcohólicas, tabaquismo, hipertensión, factores de riesgo (fuente: DeCS BIREME)

Autor corresponsal: Omar Díaz Cucho. Dirección: Pje El Divino 143- Parcona. Ica, Perú. Teléfono: (51) 056 227718. Correo electrónico: omarteroba@hotmail.com

\section{INTRODUCCIÓN}

La hiperplasia benigna de próstata (HBP) es Al crecer lo suficiente puede estrechar el la enfermedad urológica más frecuente del conducto uretral, originando los denominavarón, su prevalencia aumenta progresiva- dos síntomas del tracto urinario inferior mente con la edad. La enfermedad inicia, en (STUI): vaciamiento incompleto, polaquiuria, la mayoría de casos, entre los 40 y 50 años intermitencia, urgencia, chorro débil, pujo, de vida (1). Se reporta que entre los 51 y 60 nicturia, entre otros (4). Los cuales impactan años, el $50 \%$ de los hombres presentan HBP en la calidad de vida del paciente, convirtienhistológicamente, y en hombres mayores de do con el tiempo a la HBP en un problema 80 años, se desarrolla en más del 90\%. A los de salud pública importante (5). 75 años, el $50 \%$ de los hombres desarrollan clínicamente la enfermedad (2).

La HBP se caracteriza por un incremento en el volumen de la glándula, debido a proliferación de células estromales y epiteliales, con mayor frecuencia en la zona transicional (3).
La etiología de la HBP sigue siendo desconocida. Diversos autores han intentado describir y asociar diversos factores con el surgimiento de la HBP y con la intensidad de la sintomatología prostática. Sin embargo, el conocimiento sobre el tema sigue siendo muy controversial, lo que justifica la realización de nuevos estudios, que permitan encontrar factores de riesgo. Por lo cual, es importante conocer los factores relacionados con la intensidad de STUI en pacientes con HBP.

El objetivo del trabajo fue determinar los factores que influyen en la intensidad de los síntomas del tracto urinario inferior en pacientes con hiperplasia benigna de próstata del hospital "Augusto Hernández Mendoza” de Ica, 2011.

\section{MATERIALES Y MÉTODOS}

Se realizó un estudio transversal y correlacional en el Departamento de Ica, que se encuentra en la costa sur del territorio peruano, posee un clima cálido y seco con temperatura media de $22^{\circ} \mathrm{C}$. Tiene un total de 711,932 habitantes, de los cuales 353,386 son varones, y de éstos el $21 \%$ aproximadamente tiene más de 40 años. El 42,9\% de la población tiene seguro de salud, y el $28,2 \%$ se encuentra asegurado en la Seguridad Social del Estado (6).

Se estudió a los pacientes diagnosticados de HBP en los consultorios de urología del Hospital Essalud IV "Augusto Hernández Mendoza". Se excluyeron a los pacientes con antecedente de cirugía urológica, antecedente de neoplasia de próstata y vejiga o con trastornos psiquiátricos. Para determinar la muestra se consideró el número de pacientes atendidos por hiperplasia benigna de próstata en el servicio de urología durante el año 2010, que según la oficina de estadística del hospital fue de 1407 pacientes. Se consideró un nivel de confianza del 
95\%, una precisión del 3\%, y una prevalencia de STUI moderados/severos del $90 \%$ que encontramos en estudio piloto previo sobre 150 pacientes, dando como resultado un tamaño muestral de 302 pacientes.

Los datos se obtuvieron por muestreo no probabilístico y se utilizó un cuestionario que constó de dos partes. La primera parte recogió información referente a características sociodemográficas, tipo de dieta, hábitos noscivos y antecedentes patológicos como diabetes, hipertensión y prostatitis. La dieta se dividió en 2 grupos, tomando como modelo el estudio de Reddy y Katan: el primer grupo, dieta "prudente" incluyó un alto consumo de vegetales; el segundo grupo, dieta "occidental" incluyó un elevado consumo de carnes y grasas (7). En cuanto a hábitos noscivos, se usó la definición de bebedor abusivo de Freedman et al (8), como aquél que se embriagaba al menos una vez al mes durante el último año y, se consideró fumador, al varón que consumía al menos un cigarrillo diariamente, basándonos en el criterio de la Organización Mundial de la Salud (9).

La segunda parte de la encuesta evaluó la severidad de los síntomas del tracto urinario inferior, utilizando la versión validada al castellano del International Prostate Symptom Score (I-PSS). El I-PSS evalúa siete ítems sobre síntomas irritativos (polaquiuria, urgencia y nicturia) y obstructivos (vaciado incompleto, intermitencia, chorro débil y pujo). A cada ítem se le da una calificación del o al 5, según la frecuencia con que se presenta. De esta manera, valora la intensidad de los síntomas en tres categorías: leve (1-7 puntos), moderado (8-19 puntos) y severo (20-35 puntos). Además, posee un ítem independiente que valora la afectación de la calidad de vida del paciente, que se interpreta como: ligera (0-2 puntos), moderada (3 puntos), y grave (4-6 puntos) (10). El I-PSS está diseñado para autoadministración, no obstante, decidimos capacitar a una persona, trabajadora de salud, para la aplicación de las encuestas.

Se determinó la frecuencia, porcentaje, media y mediana para las variables cuantitativas. Se aplicó chi2 para determinar asociación entre variables cualitativas y se estimó la "Odds Ratio" (OR) junto a su intervalo de confianza al 95\%. Se realizó regresión logística para evaluar la probabilidad de tener I-PSS moderado/severo utilizando las variables: edad, nivel de instrucción, prostatitis, hipertensión, diabetes, consumo de alcohol y consumo de cigarrillos. Para realizar el análisis estadístico se utilizaron los paquetes informáticos SPSS 20 y EPIDAT 3.1.

\section{RESULTADOS}

En total fueron estudiados 382 individuos. La edad promedio fue de 68,94 años con una desviación estándar (DE) de $\pm 10,04$ años. La prevalencia de STUI moderados/severos fue de $85,1 \%$; el $84,3 \%$ eran mayores de 60 años de edad; el $47,1 \%$ eran pobladores de un área urbana; el $89 \%$ eran casados o convivientes; y el 76,9\% tenían estudios superiores (Tabla 1 ).

\begin{tabular}{|c|c|}
\hline Variable & $\mathrm{N}(\%)$ \\
\hline \multicolumn{2}{|l|}{ Edad } \\
\hline Menor de 40 años & $2(0,5)$ \\
\hline 40-49 años & $9(2,4)$ \\
\hline 50 a 59 años & $49(12,8)$ \\
\hline 60 a 69 años & $129(33,8)$ \\
\hline Mayor de 70 años & $193(50,5)$ \\
\hline \multicolumn{2}{|l|}{ Área } \\
\hline Rural & $202(52,9)$ \\
\hline Urbana & $180(47,1)$ \\
\hline \multicolumn{2}{|l|}{ Estado civil } \\
\hline Soltero & $2(0,5)$ \\
\hline Casado/conviviente & $340(89,0)$ \\
\hline Viudo & $20(5,2)$ \\
\hline Divorciado & $20(5,2)$ \\
\hline \multicolumn{2}{|l|}{ Instrucción } \\
\hline Sin instrucción & $o(0,0)$ \\
\hline Primaria & $6(1,6)$ \\
\hline Secundaria & $84(22,0)$ \\
\hline Superior & $292(76,4)$ \\
\hline \multicolumn{2}{|l|}{ Trabaja } \\
\hline Si & 175 \\
\hline No & 207 \\
\hline \multicolumn{2}{|l|}{ Ocupación } \\
\hline Agricultor & $76(19,9)$ \\
\hline Obrero & $23(6,0)$ \\
\hline Profesional/técnico & $267(69,9)$ \\
\hline Empleado de oficina & $2(0,5)$ \\
\hline Comerciante & $1(0,3)$ \\
\hline Otros & $13(3,4)$ \\
\hline
\end{tabular}

El 77\% de los participantes tenían sobrepeso; el 58,9\% manifestaban seguir actualmente una dieta a predominio de ingesta de vegetales; el 75,1\% de los participantes se habían embriagado al menos una vez al mes durante el último año; y el 78,8\% referían haber consumido al menos un cigarrillo diario durante el último año (Tabla 2 ).

99,5\% de los participantes referían tener o haber tenido algún familiar con HBP; el 46,1\% manifestaban haber sufrido de prostatitis; el $41,1 \%$ eran diabéticos; el $80,4 \%$ eran hipertensos; y el $89 \%$ de los encuestados tomaban medicación para la HBP (Tabla 3).

En la Tabla 4, se comparan las medias de las variables cuantitativas respecto a I-PSS moderado/severo frente a I-PSS leve. El síntoma más frecuente fue la polaquiuria que se
Tabla 2. Características antropométricas y estilos de vida de los participantes $(n=$

382)

\begin{tabular}{|c|c|}
\hline Variable & $N(D E)$ \\
\hline Talla (cm) & $170( \pm 6,8)$ \\
\hline Peso (Kg) & $77,87( \pm 6,2)$ \\
\hline \multirow[t]{2}{*}{ IMC (Kg/m2) } & $26,97( \pm 2,25)$ \\
\hline & $\mathrm{N}(\%)$ \\
\hline Normal $(18.5-24,9)$ & $64(16,8)$ \\
\hline Sobrepeso $(25-29,9)$ & $294(77,0)$ \\
\hline Obeso $(30-34,9)$ & $24(6,3)$ \\
\hline \multicolumn{2}{|l|}{ Dieta } \\
\hline Dieta 1 (verduras) & $225(58,9)$ \\
\hline Dieta 2 (carnes) & $157(41,1)$ \\
\hline \multicolumn{2}{|l|}{ Consumo de alcohol $^{*}$} \\
\hline Sí & $287(75,1)$ \\
\hline No & $95(24,9)$ \\
\hline \multicolumn{2}{|l|}{ Consumo de cigarrillos $^{\dagger}$} \\
\hline Sí & $301(78,8)$ \\
\hline No & $81(21,2)$ \\
\hline \multicolumn{2}{|c|}{$\begin{array}{l}\text { IMC: Índice de Masa Corporal DE: Desviación Estándar. } \\
\text { Dieta } 1 \text {. Mayor consumo de verduras, frutas, frijoles, } \\
\text { garbanzo, habas, lentejas, pollo sancochado y pescado } \\
\text { Dieta z. Mayor consumo de carnes rojas, carnes procesa- } \\
\text { das, frituras, dulces y postres, comida rápida } \\
\text { (hamburguesas, pizzas, salchipapas, etc.) } \\
\text { "Embriagarse al menos una vez al mes durante el último } \\
\text { año. } \\
{ }^{\circ} \text { Consumir al menos un cigarrillo diario en el último año. }\end{array}$} \\
\hline
\end{tabular}

Tabla 3. Antecedentes patológicos familia-

res y personales de los participantes $(\mathrm{n}=382)$

\begin{tabular}{ll}
$\begin{array}{l}\text { Variable } \\
\text { Antecedente familiar de } \\
\text { HBP }\end{array}$ & $\mathbf{N}(\%)$ \\
Sí & \\
No & $380(99,5)$ \\
¿Quién? & $2(0,5)$ \\
Padre & \\
Abuelo & $212(55,8)$ \\
Más de uno & $26(6,8)$ \\
Prostatitis & $142(37,4)$ \\
Sí & \\
No & $176(46,1)$ \\
Diabetes & $206(53,9)$ \\
Sí & \\
No & $157(41,1)$ \\
Hipertensión & $225(58,9)$ \\
Sí & \\
No & $307(80,4)$ \\
\hline HBP: Hiperplasia Benigna de Próstata & $75(19,6)$ \\
\hline
\end{tabular}

presentó en el 97,9 de los pacientes. El 77,2\% de los pacientes con I-PSS leve y el $37,2 \%$ con I -PSS moderado/severo manifestaban repercusión leve de la sintomatología sobre su calidad de vida. Mientras que el 17,5\% de pacientes con I-PSS leve y el $53,8 \%$ con I-PSS moderado/severo presentaron una repercusión moderada sobre su calidad de vida. Se estimó la probabilidad de tener STUI moderados/severos frente a STUI leves. La frecuencia de STUI moderados/severos en pacientes mayores de 60 años fue 2,63 veces mayor que en aquéllos menores de 60 años (IC95\% 1,356 -5,099). 
(IC95\% 1,356 -5,099). Los participantes con nivel de instrucción no superior tuvieron 2,45 veces más probabilidad de tener STUI moderados/severos que aquellos con educación superior (IC95\% 1,069-5,614) (Tabla 5).

Los individuos que eran diabéticos presentaron una frecuencia de 2,17 veces mayor de tener STUI moderados/severos (IC95\% 1,158$4,075)$ y los hipertensos tuvieron 2,4 veces más probabilidad de presentar STUI moderados/severos. En los pacientes con antecedente de prostatitis se encontró asociación significativa con una menor prevalencia de STUI moderados/severos (OR 0,369; IC $95 \%$ 0,203-0,667). (Tablas 6). Además, se encontró asociación inversa entre la edad y la prostatitis; siendo esta última, menos frecuente en mayores de 60 años respecto a los de menor edad ( $p$ 0,000; OR 0,165; IC $95 \%$ 0,84 $0,323)$.

Respecto a los hábitos nocivos, se encontró que los individuos que se embriagaban al menos una vez al mes durante el último año presentaron una frecuencia 4,4 veces mayor de tener STUI moderados/severo. Los que habían consumido al menos un cigarrillo diario durante el último año presentaron 3,42 veces mayor probabilidad de tener STUI moderados/severos (Tabla 7 ).

Se ajustó un modelo de regresión logística en base a la edad, nivel de instrucción, consumo de alcohol, consumo de cigarrillos y antecedentes de prostatitis, diabetes e hipertensión. La frecuencia de STUI moderados/severos fue de 3,1 veces mayor en personas que se embriagaron al menos una vez al mes durante el último año (OR 3,145; IC95\% 1,629-6,072).

\section{DISCUSIÓN}

En el Perú, en los últimos años se ha reportado un incremento en la población adulta mayor (6), por lo que se espera que la incidencia de HBP aumente. Por lo que, es importante conocer los factores que influyen en la intensidad de los STUI en pacientes con HBP. En este estudio encontramos que, en pacientes del Hospital IV Essalud "Augusto Hernández Mendoza” de Ica, la edad, el nivel bajo de estudios, la hipertensión, la diabetes, el consumo de alcohol y cigarrillos se relacionan con STUI más severos.

Aunque la prevalencia de STUI moderados/ severos $(85,1 \%)$ es similar a la encontrada por Saldaña en el Hospital Nacional Arzobispo Loayza de Lima (11) y por Li et al (12), en Asia, supera enormemente a la reportada por Fernández et al (13), en España (16,6\%). Además, encontramos que la prevalencia de STUI aumenta con la edad, lo cual es compatible con
Tabla 4. Diferencia de medias respecto a STUI moderados/severos frente a STUI leves en los participantes $(n=382)$

\begin{tabular}{|c|c|c|c|c|c|c|c|c|c|c|c|}
\hline \multirow[b]{2}{*}{ Edad } & \multicolumn{3}{|c|}{$\begin{array}{c}\text { Total } \\
\text { Media } \pm \text { DE }\end{array}$} & \multicolumn{3}{|c|}{$\begin{array}{r}\text { I-PSS } \geq 8 \\
(n=325)\end{array}$} & \multicolumn{3}{|c|}{$\begin{array}{c}\text { I-PSS }<8 \\
(n=57)\end{array}$} & \multirow{2}{*}{$\begin{array}{c}t \\
-1,572\end{array}$} & \multirow{2}{*}{$\begin{array}{c}P \\
0,117\end{array}$} \\
\hline & 68,94 & \pm & 10,04 & 69,28 & \pm & 9,73 & 67,02 & \pm & 11,6 & & \\
\hline Peso & 77,87 & \pm & 6,207 & 77,97 & \pm & 6,18 & 77,3 & \pm & 6,37 & $-0,756$ & 0,45 \\
\hline Talla & 1,701 & \pm & 0,68 & 1,702 & \pm & 0,068 & 1,692 & \pm & $\begin{array}{l}0,06 \\
4\end{array}$ & $-1,033$ & 0,302 \\
\hline IMC & 26,97 & \pm & 2,25 & 26,96 & \pm & 2,24 & 27,04 & \pm & 2,38 & 0,267 & 0,79 \\
\hline Síntomas & 5,51 & \pm & 1,14 & 5,82 & \pm & 0,88 & 3,74 & \pm & 0,74 & $-16,816$ & $<0,000$ \\
\hline $\begin{array}{l}\text { Puntaje } \\
\text { I-PSS }\end{array}$ & 10,64 & \pm & 3,07 & 11,51 & \pm & 2,4 & 5,7 & \pm & 1,25 & $-7,806$ & $<0,000$ \\
\hline
\end{tabular}

Tabla 5. Análisis univariado de los factores sociodemográficos asociados a la prevalencia de STUI moderados/severos frente a STUI leves

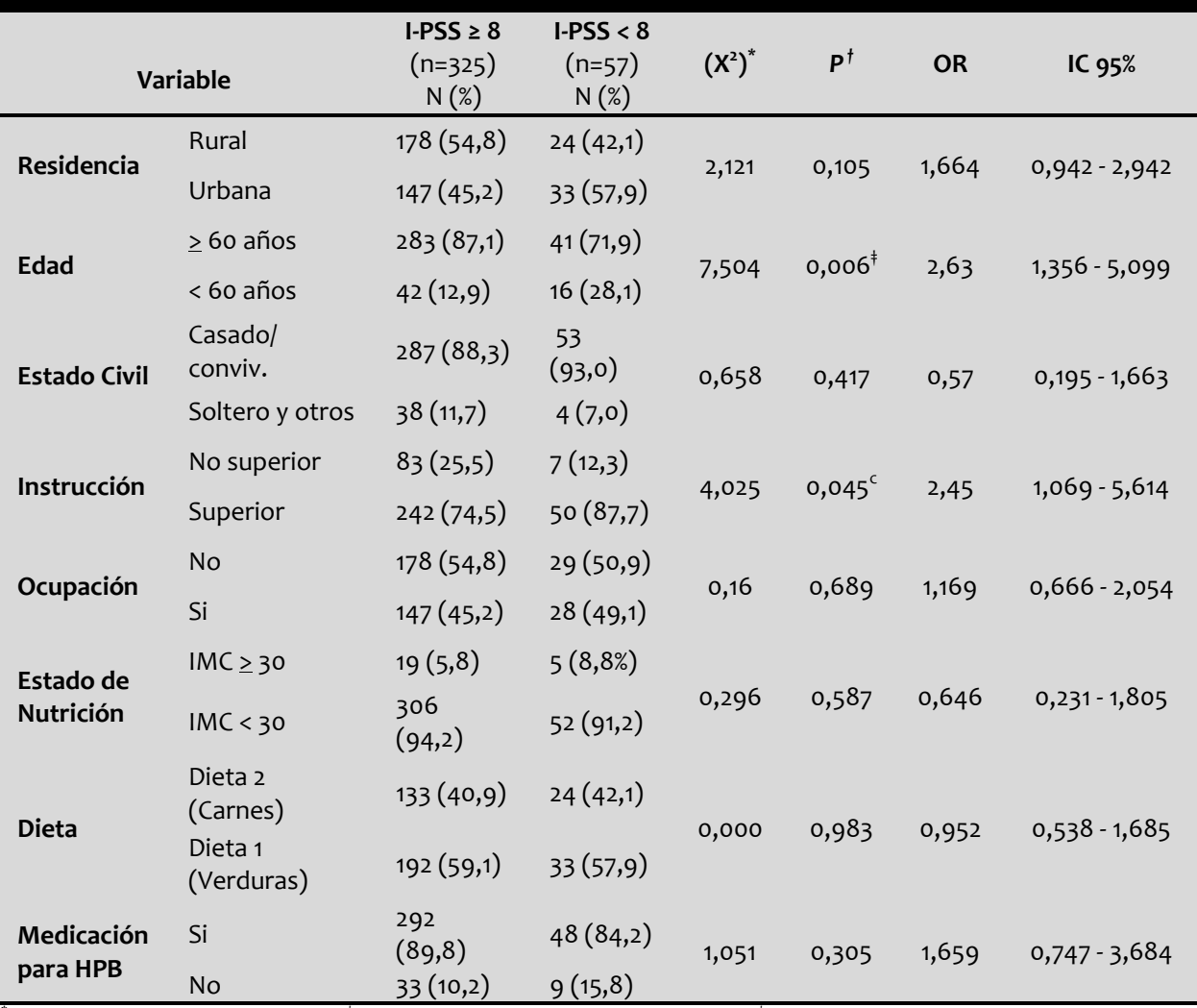

Corrección de Yates (grado de libertad 1). ${ }^{\dagger}$ Valor $p$ calculado para la prueba exacta de Fisher. ${ }^{\ddagger}$ Evidencia diferencia significativa $(p<0.05)$.

Dieta 1. Mayor consumo de verduras, frutas, frijoles, garbanzo, habas, lentejas, pollo sancochado y pescado

Dieta 2.- Mayor consumo de carnes rojas, carnes procesadas, frituras, dulces y postres, comida rápida (hamburguesas, pizzas, salchipapas, tc.)

Tabla 6. Análisis univariado de los antecedentes personales y familiares asociados a la prevalencia de STUI moderados/severos frente a STUI leves.

\begin{tabular}{|c|c|c|c|c|c|c|c|}
\hline \multicolumn{2}{|l|}{ Variable } & $\begin{array}{c}\text { I-PSS } \geq 8 \\
(n=325) \\
N(\%)\end{array}$ & $\begin{array}{c}\text { I-PSS < } 8 \\
(\mathrm{n}=57) \\
\mathrm{N}(\%)\end{array}$ & $\left(X^{2}\right)^{*}$ & $\mathbf{P}^{\dagger}$ & OR & IC 95\% \\
\hline \multirow{2}{*}{$\begin{array}{l}\text { Diabetes } \\
\text { Mellitus }\end{array}$} & $\mathrm{Si}$ & $142(43,7)$ & $15(26,3)$ & \multirow[b]{2}{*}{5,352} & \multirow{2}{*}{$0,021^{c \ddagger}$} & \multirow[b]{2}{*}{2,173} & \multirow[b]{2}{*}{$1,158-4,075$} \\
\hline & No & $183(56,3)$ & $42(73,7)$ & & & & \\
\hline \multirow{2}{*}{$\begin{array}{l}\text { Hipertensión } \\
\text { Arterial }\end{array}$} & Si & $269(82,8)$ & $38(66,7)$ & \multirow[b]{2}{*}{6,981} & \multirow{2}{*}{$0,008^{c}$} & \multirow[b]{2}{*}{2,401} & \multirow[b]{2}{*}{$1,290-4,471$} \\
\hline & No & $56(17,2)$ & $19(33,3)$ & & & & \\
\hline \multirow[t]{2}{*}{ Prostatitis } & Si & $138(42,5)$ & $38(66,7)$ & \multirow[b]{2}{*}{10,482} & \multirow{2}{*}{$0,001^{c}$} & \multirow[b]{2}{*}{0,368} & \multirow[b]{2}{*}{$0,203-0,667$} \\
\hline & No & $187(57,5)$ & $19(33,3)$ & & & & \\
\hline \multirow{2}{*}{$\begin{array}{l}\text { Antec. } \\
\text { familiares }\end{array}$} & Si & $323(99,4)$ & $57(100)$ & \multirow{2}{*}{0} & \multirow[b]{2}{*}{1} & \multirow{2}{*}{---} & \multirow{2}{*}{--} \\
\hline & No & $2(0,6)$ & $0(0,0)$ & & & & \\
\hline
\end{tabular}

“Corrección de Yates (grado de libertad 1). ${ }^{\dagger}$ Valor $p$ calculado para la prueba exacta de Fisher. ${ }^{\dagger}$ Evidencia diferencia significativa ( $\left.p<0.05\right)$. 
aumenta con la edad, lo cual es compatible con los hallazgos de otros estudios (13-16). Se plantea que la asociación entre edad avanzada y HBP se debe al desequilibrio entre proliferación celular y apoptosis. Con el paso de los años la próstata crece, pero los andrógenos periféricos disminuyen. Sin embargo, se conserva un nivel elevado de dihidrotestosterona (DHT) a nivel prostático, que proviene del metabolismo de la testosterona, mediado por la enzima 5 alfa reductasa tipo 2 (14). La DHT y la testosterona se unen a los receptores nucleares de andrógenos en las células estromales y epiteliales de la próstata, induciendo factores de crecimiento que actúan sobre la misma glándula. La DHT es 10 veces más potente que la testosterona (15). Así mismo, en la edad avanzada se conserva un nivel alto de receptores de andrógenos, que son inducidos por niveles altos de estrógenos, lo que haría a la próstata más sensible a los efectos androgénicos (2).

Pocos estudios han valorado la influencia de las características sociodemográficas en los STUI $(13,16)$. Un estudio de cohorte prospectivo que incluyó a 21,949 participantes en EEUU encontró que el tipo de seguro, el tiempo de la última visita al médico, el nivel de educación y el matrimonio tienen asociación con el desarrollo de HBP (16). Nosotros encontramos relación de manera independiente solo con el nivel de estudios. Pero creemos, que aquellos factores que estén relacionados con un acceso adecuado a la salud, tales como, la cobertura de seguro, el ingreso económico, el estado civil, el nivel de educación entre otros, contribuyen a la posibilidad de un diagnóstico temprano de HBP. Por el contrario, si el acceso a la salud es deficiente habrá un diagnóstico tardío de HBP y por ende STUI más severos.

Actualmente hay datos contradictorios sobre la asociación entre el consumo de alcohol y STUI. Al parecer en los hombres, el consumo de alcohol muestra una relación de curva en forma de "J" con el riesgo moderado a severo de STUI como la descrita por Oh et al (17), en un estudio transversal realizado en 30,196 varones de Corea del Sur, encontrando un OR de STUI moderados/severos de 0,91(IC95\% 0,84-0,98) en hombres que bebían o a $10 \mathrm{~g}$ por día y 1,19(IC95\% 1,07-1,33) en aquellos que bebían $40 \mathrm{~g}$ o más diariamente. Nosotros encontramos que los participantes que manifestaban haberse embriagado al menos una vez al mes durante el último año, presentan 4 veces más frecuencia de STUI moderados/ severos que aquellos con ingesta menor o no consumo de alcohol (OR 4,44; IC $95 \%$ 2,477,99).

Tabla 7. Análisis univariado de los hábitos nocivos asociados a la prevalencia de STUI moderados/ severos frente a STUI leves.

\begin{tabular}{|c|c|c|c|c|c|c|c|}
\hline Variable & & $\begin{array}{c}\text { I-PSS } \geq 8 \\
(n=325) \\
N^{\circ}(\%)\end{array}$ & $\begin{array}{c}\text { I-PSS < } 8 \\
(n=57) \\
N^{\circ}(\%)\end{array}$ & $\left(X^{2}\right)^{*}$ & $p^{\dagger}$ & OR & IC 95\% \\
\hline Alcohol & $\begin{array}{l}\mathrm{Si} \\
\mathrm{No}\end{array}$ & $\begin{array}{c}260(80,0) \\
65(20,0)\end{array}$ & $\begin{array}{l}27(47,5) \\
30(52,6)\end{array}$ & 25,918 & $<0,00 \ddagger$ & 4,444 & $2,471-7,991$ \\
\hline Tabaco & $\begin{array}{l}\mathrm{Si} \\
\text { No } \\
\end{array}$ & $\begin{array}{c}268(82,5) \\
57(17,5) \\
\end{array}$ & $\begin{array}{l}33(57,9) \\
24(42,1) \\
\end{array}$ & 16,078 & $<0,00 \neq$ & 3,419 & $1,879-6,221$ \\
\hline
\end{tabular}

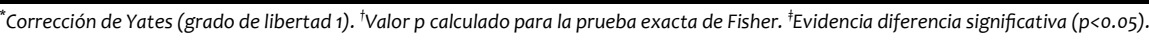

Además, esta asociación persistió al realizar regresión logística (OR 3,15; IC95\% 1,63-6,07), controlando por edad, nivel de instrucción, diabetes, hipertensión, consumo de cigarrillos y prostatitis. Este efecto adverso del alcohol sobre STUI podría deberse a que una ingesta excesiva de alcohol, aproximadamente $>50 g /$ día, estimula la actividad del sistema nervioso simpático, lo que produce aumento del tono del musculo liso prostático (18). Además, el alcohol tiene varios efectos sistémicos, incluyendo incremento de la diuresis, exacerbando así a los STUI (19).

Los resultados de nuestro estudio permiten plantear la hipótesis de que no es necesario un consumo continuo de alcohol en exceso para empeorar la sintomatología del HBP. Para esclarecer este punto, recomendamos realizar otros estudios que evalúen con más detalle la frecuencia de ingesta, cantidad y tipo de alcohol. El efecto protector del alcohol sobre HBP, reportado por otros autores, se explicaría por disminución del agrandamiento de la próstata, debido a que el consumo moderado de alcohol produce depresión transitoria de los niveles de testosterona plasmática (18).También se ha planteado que mejora la sensibilidad a la insulina (20). Por otro lado, podría deberse a que el consumo moderado de alcohol tiene efecto beneficioso sobre los trastornos metabólicos relacionados con la salud cardiovascular. Las perturbaciones metabólicas ocasionan alteración microvascular e inflamación, produciendo a su vez, isquemia y estrés oxidativo, los cuales se relacionan con el surgimiento de HBP (19). Pero esto, no es del todo incompatible con nuestros hallazgos, ya que nosotros estudiamos el efecto del alcohol sobre STUI y no directamente sobre HBP. Varios autores, plantean que el alcohol disminuye el riesgo de HBP posiblemente por los mecanismos ya explicados, pero aumenta la severidad de los STUI, debido a que los STUI no solo se originan en la próstata sino también en la vejiga. Así, el alcohol exacerbaría sobre todo los STUI originados en la vejiga (19).

En este estudio, los varones que consumieron al menos un cigarrillo diario durante el último año fueron 3 veces más propensos de presen- tar STUI moderados/severos, lo que coincide con otros estudios que han encontrado evidencias de un aumento de intensidad de los STUI con el tabaquismo $(13,21)$. La asociación del tabaquismo con los STUI en HBP es muy controversial. Los mecanismos exactos de porqué el fumar aumenta el riesgo de STUI son desconocidos, pero podría deberse a que la nicotina incrementa la actividad del sistema nervioso simpático, aumentando el tono de la próstata y del músculo liso vesical, exacerbando los STUI, principalmente los de tipo irritativos $(18,20,22)$. Por otro lado, se describe en los fumadores, un aumento en la concentración de hormonas sexuales, por lo que los niveles altos de testosterona, y principalmente dihidrotestosterona, estimularían el crecimiento prostático y el desarrollo de STUI en HBP $(18,23)$.

En contraste, otros estudios describen un efecto protector con niveles moderados de consumo de cigarrillos $(24,25)$, que podría deberse a la asociación existente del hábito de fumar con la disminución del apetito y de tejido adiposo, lo que provocaría un mecanismo de modulación del peso corporal (26). Sin embargo, cualquier efecto protector proporcionado parece ser dosis dependiente, como se grafica en el estudio de Fernández et al, quienes describen una asociación bifásica entre tabaquismo y STUI, encontrando entre la población de fumadores de 1 a 34 cigarrilIos/día una prevalencia de STUI moderados/ severos menor respecto a los no fumadores, y resaltando una mayor prevalencia de STUI moderados/severos a partir del consumo de más de 34 cigarrillos/día (13).

Esta relación bifásica podría deberse al doble efecto que puede ocasionar la nicotina a nivel de los receptores parasimpáticos postsinápticos ubicados en la vejiga, ejerciendo un rol agonista a concentraciones bajas y antagonista a concentraciones altas; con lo que dosis bajas mejorarían la función de la vejiga, mientras las altas dificultarían la micción (25).

Las prevalencias de hipertensión arterial (HTA) y HBP aumentan con la edad. Por lo que, ambas enfermedades son comunes en varones de edad avanzada. Se reporta que 
del 25 al 30\% de los hombres mayores de 60 años con HBP sufren concomitantemente de $\operatorname{HTA}(24,27)$.

En nuestro estudio encontramos una prevalencia muy superior de HTA en pacientes con HBP, de aproximadamente $80,4 \%$. Además, los hipertensos resultaron tener 2 veces más probabilidad de presentar STUI moderados/ severos (OR: 2,40; IC95\%1,29 - 4,47) que aquellos que no lo son. Lo que confirma los resultados de otros estudios $(13,27,28)$. Como el realizado por Fernández et al, en España, sobre 1806 pacientes, donde reportan que los hipertensos tienen 1,77 veces más probabilidad de presentar STUI severos (IC95\%1,36-2,29) que los no hipertensos (13).

La asociación entre HTA y HBP se debería a la actividad del sistema nervioso simpático, que regula tanto el tono arterial como la fisiología miccional $(28,29)$. El estimulo simpático probablemente tenga efecto sobre el crecimiento prostático al enlentecer la apoptosis (29). Además, se sabe que los niveles de catecolaminas están elevados en pacientes hipertensos $(28,29)$. Las catecolaminas pueden aumentar el tono del musculo liso prostático y empeorar la obstrucción al vaciamiento de la vejiga. Los niveles de catecolaminas son más altos durante el día y disminuyen en la noche por lo que, puede haber menor diuresis en el día, por tanto, mayor retención de orina. Durante la noche la diuresis aumentaría por descenso de catecolaminas y al producirse mayor flujo renal.

Así, la nicturia es mayor en pacientes hipertensos (28). Por otro lado, se ha informado de hiperactividad del sistema renina angiotensina aldosterona a través de la angiotensina II, un potente vasoconstrictor, estimulador de la proliferación celular y facilitador del impulso simpático. La angiotensina II, también, afectaría el tono del músculo liso prostático y del músculo detrusor de la vejiga a través de sus receptores ubicados en la próstata y la vejiga, respectivamente (27).

La asociación entre diabetes y HBP ha sido estudiada, aunque unos pocos investigadores no describen asociación (24, 30). Nosotros encontramos que los varones con diabetes presentan una frecuencia 2 veces mayor de STUI moderados/severos en HBP que los no diabéticos. Estudios previos señalan que la diabetes no solo se asocia con un mayor riesgo de presentar HBP sino también con presentar mayor severidad en los síntomas $(26,31,32)$, lo que refuerza nuestro hallazgo. Por ejemplo, Sarma et al, encontraron que los varones con diabetes fueron 1,28 veces más propensos a reportar STUI moderados/ severos (31). Varios mecanismos se han planteado para establecer la influencia de la diabetes sobre la HBP. Ambas patologías pueden provocar sintomatología urológica similar, afectando la función de la vejiga y produciendo síntomas obstructivos e irritativos, pero mediante fisiopatología distinta aún no conocida totalmente (32).

La diabetes se relacionaría con daño en la función del detrusor, mientras que en HBP no existiría daño directo en la función del detrusor sino más bien alteración en los componentes físicos estáticos y dinámicos que participan en el aumento de la resistencia a la salida del contenido de la vejiga; ambos mecanismos conllevan a una tasa máxima de flujo reducida y un aumento del volumen residual posterior a la evacuación (33). El mal control de la diabetes puede causar diuresis osmótica que se asocia con una mayor frecuencia urinaria y nicturia (34). Por otro lado, la insulina es una hormona del crecimiento que estimula el desarrollo y la reproducción celular (35). Se plantea que el aumento de insulina produce aumento en el número de receptores de hormona del crecimiento a nivel hepático, que a su vez estimula la producción hepática del factor de crecimiento parecido a insulina tipo 1 (IGF-1) promoviendo la proliferación de células prostáticas (36). El aumento de nivel de insulina se relaciona también, con un mayor metabolismo de glucosa a nivel del núcleo ventromedial del hipotálamo que regula el sistema nervioso simpático, aumentando la producción de receptores adrenérgicos de la próstata y las catecolaminas, lo que se sugiere produce un efecto trófico en la próstata al retardar el proceso apoptótico (35). Además, el estimulo simpático aumenta la contracción del músculo liso de la próstata y el cuello vesical, incrementando así la severidad de STUI (37).

Varios estudios evidencian relación directa entre prostatitis y los STUI $(38,39)$. Sauver et al, en un estudio longitudinal sobre 2447 varones en EEUU, que siguieron durante 14 años, encontraron que la prostatitis se asoció a una probabilidad 2,4 veces mayor de desarrollar HBP, y concluyen que la prostatitis puede ser un marcador temprano o un factor de riesgo de desarrollar problemas prostáticos o urológicos (39).

En nuestro estudio se encontró una asociación inversa, los STUI moderados/severos se presentaron con menor frecuencia en pacientes con antecedente de prostatitis, esto podría deberse a que también encontramos asociación inversa entre prostatitis y la edad. Por lo que, el antecedente de prostatitis, al presentarse con mayor frecuencia en varones menores de 60 años, hace que se relacione también, con mayor frecuencia a STUI leves que a STUI moderados/severos. Por tanto, la edad podría estar actuando como factor confusor. Sin embargo, esto no descarta el papel de la prostatitis en la patogenia del HBP. Se sabe que, la acción generada por agentes infecciosos puede provocar, en las células estromales, la liberación de sustancias proinflamatorias e inducir el crecimiento prostático (40).

Dentro de las limitaciones de nuestro estudio, tenemos que el 99,5\% de pacientes refería antecedente familiar de HBP, pero no se pudo calcular el OR, ya que la muestra de pacientes sin hipertensión fue insuficiente. Por otro lado, abordamos la influencia de hipertensión y diabetes en la intensidad de STUI sin tomar en cuenta la medicación recibida para estas patologías, pero, consideramos que la influencia del tratamiento de la hipertensión y la diabetes sería mejor abordada en un estudio más amplio. Las cantidades específicas de ingesta de alcohol y cigarrillos no se tomaron en cuenta en este estudio, porque, consideramos que deberían ser mejor evaluadas en otro estudio que aborde además, la influencia del tipo de alcohol o tabaco. Nosotros evaluamos el efecto sobre STUI en el bebedor abusivo y en el fumador, encontrando significancia estadística, lo que no le resta importancia a nuestros hallazgos.

Por último, dada la alta prevalencia de HTA asociado a HBP en el Hospital Essalud "Augusto Hernández Mendoza" de Ica, recomendamos a urólogos, cardiólogos y médicos internistas reconocer la frecuente coexistencia de estas dos patologías al momento de tomar una decisión sobre manejo terapéutico. Además, los Hospitales deberían prepararse para atender una mayor demanda de pacientes con HBP.

\section{REFERENCIAS BIBLIOGRÁFICAS}

1. Fernández $\mathbf{M}$, Pereira I. Hiperplasia benigna de próstata: una afección de elevada prevalencia en el paciente de edad avanzada. Rev Esp Geriatr Gerontol. 2008; 43(1): 44-51.

2. Presti J, Kane C, Shinohara K, Carroll P. Neoplasms of the prostate gland. En: Tanagho E, McAninch J, editores. Smith's general urology, $17^{\text {a }}$ ed. Nueva York: Mc Graw Hill Lange. 2008. pp.348-74.

3. Algaba F, Trias I, López L, Rodríguez JM, González J. Evolución morfológica de la Hiperplasia de próstata. Actas Urol Esp. 2000; 24(6):463-67.

4. Ruiz Ramos $\mathbf{M}$, et al. Consenso sobre terminología y conceptos de la función del tracto urinario inferior. Grupo Español de Urodinámica y de SINUG. Actas Urol Esp. 
2005; 29(1):16-30.

5. Bobé $F$, Buil $M$, Allué $A$, Morro A, Maxenchs $\mathbf{M}$, Gens $\mathbf{M}$. Hiperplasia benigna de próstata. Necesidad de valorar la calidad de vida en el proceso terapéutico. Aten Primaria. 2006; 38(7):387-91.

6. Instituto Nacional de Estadística e Informática. Censos Nacionales 2007: XI de Población y VI de Vivienda. Lima: Instituto Nacional de Estadística e Informática 2007. Disponible en: http://censos.inei.gob.pe/ censos2007

7. Reddy KS, Katan MB. Diet, Nutrition and the Prevention of Hypertension and Cardiovascular Diseases. Public Health Nutrition. 2004; 7(1A):167-186. Disponible en: http://www.who.int/nutrition/publications/ public_health_nut5.pdf

8. Freedman A, Kaplan H, Sadock B. Modern synopsis of comprehensive text book of Psychiatry. 2 ed. Baltimore: Williams and Wilkins.1977:p. 676.

9. World Health Organization. Guidelines for controlling and monitoring the tobacco epidemic. Geneva: Tobacco or Health Programme, WHO, 1997

10. Vela R, Martín JM, Calahorra J, Damián J, Hernández A, Boyle P. Validación cultural y lingüística en castellano del baremo internacional de síntomas prostáticos (IPSS). Act Urol Esp. 1994; 18:841-7.

11. Saldaña J. Prevalencia de vejiga hiperactiva en pacientes con diagnóstico clínico de hiperplasia prostática benigna en el Hospital Nacional Arzobispo Loayza. [Tesis de postgrado] Lima: Unidad de postgrado, Universidad Nacional Mayor de San Marcos; 2004.

12. Li MK, García L, Patron N, Moh LC, Sundram M, Leungwattanakij S, Pripatnanont C, Cheng C, Chi-Wai M, Loi-Cheong N. An Asian multinational prospective observational registry of patients with benign prostatic hyperplasia, with a focus on comorbidities, lower urinary tract symptoms and sexual function. BJU Int. 2008; 101(2):197-202.

13. Fernández C, Moreno J, Cano S, Fuentes M, Bocardo G, Silmi A. Prevalencia de síntomas del tracto urinario inferior relacionados con la hiperplasia benigna de próstata. Estudio de 1804 hombres de 40 años o más residentes en Madrid durante 1999-2000. Actas Urol Esp. 2009; 33(1):4351

14. Roehrborn CG, Mc Conneil JD. En: Walsh Retik, Vaugham Weim editors. Campbel Urología: Etiología, fisiopatología, epidemiología y evolución natural de la hiperpla-

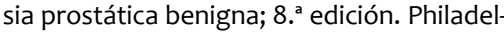
phia. Ed Panamericana. 2004, p.1417-1455.

15. Epstein J. Tracto urinario inferior y sistema genital masculino. En: Kumar V, Abbas A, Fausto $\mathrm{N}$, editores. Robbins y Cotran, patología estructural y funcional. $7^{\mathrm{a}}$ ed. Madrid: Elsevier; 2005.p.1025-1061.

16. Fowke JH, Murff HJ, Signorello LB, Lund L, Blot WJ. Race and socioeconomic status are independently associated with benign prostatic hyperplasia. J Urol. 2008; 180 (5):2091-6.
17. Oh MJ, Eom CS, Lee HJ, Choi HC, Cho B, Park JH. Alcohol consumption shows a Jshaped association with lower urinary tract symptoms in the general screening population. J Urol. 2012; 187(4):1312-7.

18. Platz EA, Rimm EB, Kawachi I, Colditz GA, Stampfer MJ, Willett WC, Giovannucci E. Alcohol consumption, cigarette smoking, and risk of benign prostatic hyperplasia. Am J Epidemiol. 1999; 149(2):106-15.

19. Parsons J, Im R. Alcohol consumption is associated with a decreased risk of benign prostatic hyperplasia. J Urol. 2009; 182(4): 1463-1468.

20. Rohrmann S, Crespo CJ, Weber JR, Smit E, Giovannucci E, Platz EA. Association of cigarette smoking, alcohol consumption and physical activity with lower urinary tract symptoms in older American men: findings from the third National Health And Nutrition Examination Survey. BJU Int. 2005; 96(1):77-82.

21. Seim A, Hoyo C, Ostbye T, Vatten L. The prevalence and correlates of urinary tract symptoms in Norwegian men: the HUNT study. BJU Int. 2005; 96(1):88-92.

22. Narkiewicz K, van de Borne PJ, Hausberg M, Cooley RL, Winniford MD, Davison DE, et al. Cigarette smoking increases sympathetic outflow in humans. Circulation. 1998; 98(6):528-34.

23. Carson C 3rd, Rittmaster R. The role of dihydrotestosterone in benign prostatic hyperplasia. Urology. 2003; 61(4Suppl1):27.

24. Meigs JB, Mohr B, Barry MJ, Collins MM, McKinlay JB. Risk factors for clinical benign prostatic hyperplasia in a communitybased population of healthy aging men. J Clin Epidemiol. 2001; 54(9):935-944

25. Roberts RO, Jacobsen SJ, Rhodes T, Guess HA, Girman CJ, Panser LA, et al. Cigarette smoking and prostatism: a biphasic association? Urology. 1994; 43(6):797-801.

26. Parsons JK. Modifiable Risk Factors for Benign Prostatic Hyperplasia and Lower Urinary Tract Symptoms: New Approaches to Old Problems. J. Kellogg Parsons. J Urol. 2007; 178(2):395-401.

27. Ito $\mathbf{H}$, Taga $\mathbf{M}$, Tsuchiyama $\mathrm{K}$, Akino $\mathbf{H}$, Yokoyama O.IPSS is Lower in Hypertensive Patients Treated With Angiotensin-II Receptor Blocker: Posthoc Analyses of a Lower Urinary Tract Symptoms Population Neurourol Urodyn. 2013 Jan; 32(1):70-4.

28. Sugaya K, Kadekawa K, Ikehara A, Nakayama T, Gakiya M, Nashiro F. Influence of hypertension on lower urinary tract symptoms in benign prostatic hyperplasia. Int J Urol. 2003; 10(11):569-74.

29. Briganti A, Capitanio U, Suardi N, Gallina A, Salonia A, Bianchi $M$, et al. Benign Prostatic Hyperplasia and Its Aetiologies. European Urology Supplements. 2009; 8:865-871.

30. Hong J, Kwon S, Yoon H, Lee H, Lee B, Kim $\mathrm{HH}$, et al. Risk factors for benign prostatic hyperplasia in South Korean men. Urol Int. 2006; 76(1):11-9.

31. Sarma AV, Burke JP, Jacobson DJ, McGree ME, St Sauver J, Girman CJ, et al. Associa- tions between diabetes and clinical markers of benign prostatic hyperplasia among community-dwelling Black and White men. Diabetes Care. 2008; 31(3):476-82.

32. Stamatiou K, Lardas M, Kostakos E, Koutsonasios V, Michail E. The Impact of Diabetes Type 2 in the Pathogenesis of Benign Prostatic Hyperplasia: A Review. Adv Urol. 2009.

33. Hammarsten J, Hogstedt B, Holthuis N, MellstromD. Components of the metabolic syndrome-risk factors for the development of benign prostatic hyperplasia. Prostate Cancer Prostatic Dis. 1998; 1 (3):157-162.

34. Menendez V, Cofan F, Talbot-Wright R, Ricart MJ, Gutierrez R, Carretero P. Urodynamic evaluation in simultaneous insulindependent diabetes mellitus and end stage renal disease. J.Urol.1996; 155 (6):2001-4.

35. Kasturi S, Russell S, McVary KT. Metabolic syndrome and lower urinary tract symptoms secondary to benign prostatic hyperplasia. Curr Urol Rep. 2006; 7(4):288-92.

36. Platz EA, Pollak MN, Leitzmann MF, Stampfer MJ, Willett WC, Giovannucci E. Plasma insulin-like growth factor-1 and binding protein-3 and subsequent risk of prostate cancer in the PSA era. Cancer Causes Control. 2005; 16(3):255-62.

37. Guess HA. Benign Prostatic Hyperplasia and Prostate Cancer. Epidemiol Rev. 2001; 23(1):152-8.

38. Chung JH, Yu JH, Sung LH, Noh CH, Chung JY. Effect of Prostatitis on Lower Urinary Tract Symptoms: Retrospective Analysis of Prostate Biopsy Tissue Korean J Urol. 2012; 53(2):109-13.

39. Sauver J, Jacobson D, McGree M, Girman C, Lieber M, Jacobsen S. Longitudinal association between prostatitis and development of benign prostatic hyperplasia. Urology. 2008; 71(3):475-479.

40. Nickel JC. Inflammation and benign prostatic hyperplasia. Urol Clin North Am. 2008; 35(1):109-115. 
FINANCIAMIENTO: Autofinanciado

CONTRIBUCIONES DE AUTORÍA: ODC, MAH y

AMA participaron en el diseño del estudio, el análisis de los datos, revisaron críticamente el artículo y aprobaron la versión final.

\title{
Factors that affect the intensity of the lower urinary tract symptoms in patients with benign prostatic hyperplasia
}

\begin{abstract}
bjective: To determine the factors that influence the severity of lower urinary tract symptoms
(LUTS ) in patients with benign prostatic hyperplasia in a hospital in Peru . Materials and methods: A cross-sectional study in 2011 on 382 men treated at the Hospital IV " Augusto Mendoza Hernández " of Ica. Through survey collected sociodemographic information and intensity of LUTS was determined by the International Prostate Symptoms Score. Chi square ( X2 ) was used to assess association between qualitative variables and the odds ratio (OR ) was estimated. Logistic regression was performed to assess the probability of having LUTS moderate / severe . Results: The prevalence of moderate / severe LUTS was 85.1 $\% .41 .1 \%$ were diabetic and $80.4 \%$ were hypertensive. Greater than or equal to 60 years , compared with less than 60 years old, presented 2.63 times more frequently than moderate / severe ( $95 \% \mathrm{Cl} 1.35$ to 5.09 ) LUTS and the low level of education, compared to high (OR $2.45,95 \% \mathrm{Cl} 1.06$ to 5.61 ), diabetes (OR 2.17, $95 \% \mathrm{Cl} 1.15$ to 4.07 ), hypertension (OR 2.4 , $95 \% 1,29-4,47$ ), excessive intake of alcohol at least once a month (OR $4.44,95 \% \mathrm{Cl} 2.47$ to 7.99 ) and consumption of at least one cigarette a day (OR $3.42 ; 95 \% \mathrm{Cl} 1.87$ to 6.22 ). Prostatitis was associated with a lower prevalence of LUTS moderate / severe (OR $0.36,95 \% \mathrm{Cl}$ 0.20 to 0.66 ). Conclusions: age, low education, hypertension, diabetes, alcohol and cigarettes were associated with more severe LUTS.
\end{abstract}

Key words: Prostatic hyperplasia, urinary tract, alcohol drinking, smoking, hypertension, risk factors . (source: MeSH NLM)

\section{CITA SUGERIDA.}

Díaz-Cucho O, Aparcana-Huayanca M, Morales-Alvarez A. Factores que influyen en la intensidad de los síntomas del tracto urinario inferior en pacientes con hiperplasia benigna de próstata. Rev méd panacea.2013; 3 (2):32-38. 